\title{
DIAGNOSING ALZHEIMER'S DISEASE AND MILD COGNITIVE IMPAIRMENT WITH MODALITIES: A SURVEY
}

\author{
S.Ieswaria ${ }^{1}$ and Dr.M.Nandhini ${ }^{2}$
}

Abstract-The effective and accurate diagnosing of Alzheimer's Disease(AD) and its previous stage, Mild Cognitive Impairment(MCI) is necessary as there's no cure for the disease and if found in early stages can be used to improve the quality of life of a person. Various modalities have been used as input for the diagnosis by various researchers to propose a computer aided diagnosis model that has high prediction and classification accuracy. The choice of input and the methodologies used in the model plays an important role in obtaining the classification accuracy of the diseases. so a survey has been made to gain knowledge about some of the existing works focusing on the modalities used.

Keywords-Alzheimer's disease, Mild Cognitive Impairment, Modalities, Feature representation and extraction, Feature selection, Classification

\section{INTRODUCTION}

Alzheimer's disease affects the brain $70 \%$ due to genes and the remaining by head injury, depression and hypertension. The disease progresses with plaques and tangles showing the symptoms; short term memory loss as main and language problem, disorientation, mood swings etc., as side effects. As it has no cure, early diagnosis is necessary to prevent the risk of progression.

The need for more accurate diagnosis has led to various research works in this field. According to the study understanding the inner details of the modalities may bring up more accurate diagnosis of the diseases. The diagnosis of the brain needs a clearer input that should provide thorough understanding of various parts of the brain especially the regions that are affected by the diseases, thus the survey has been made by grouping them based on the modalities.

Some of the modalities that are sensitive to AD/MCI diagnosis:

- Magnetic Resonance Imaging(MRI) : $\quad$ Brain atrophy measurement is given by Structural and functional MRI.

- Cerebrospinal Fluid(CSF)

- Blood Samples

- Positron Emission Tomography(PET) PET.

- Electro Encephalogram(EEG)

- Genetic information accuracy to the diagnosis.

CSF gives the quantification of proteins.

The protein content in the body is measured from the blood samples.

The glucose content is measured with PET and Fluorodeoxyglucose

$:$

: $\quad$ EEG is used to analyse the brain activity.

: The main cause of the disease is by genes, including this will give more

Chris Hinrichs et al [10], in their paper have discussed about the importance of setting the direction of researches in finding the image -based techniques for understanding the patterns during the early signs of disease's symptoms. In this paper, they have used MRI and FDG-PET for their proposal. Daoqiang Zhang et al [29], in their paper have showed the importance of having multi modalities as features for a better diagnosis accuracy.

This survey paper has been made to enlighten the modalities that are sensitive to AD/MCI. The rest of the paper is organized with the explanation about features, the list of features used in the diagnosis in table-1, the steps of classification process and tools used are discussed in section II. The surveyed works have been grouped by the modalities with explanation about each modality, the knowledge gained from the survey has been tabulated with methodologies and accuracy obtained and the result of comparison of the surveyed works in the table- 2 has been is conveyed in section III. The conclusion of the survey work is given in section IV followed by the reference papers.

\section{CLASSIFICATION PROCESS}

The system works by pre-processing the input modalities and extraction of essential features forming an optimal subset selection for better prediction and classification between $\mathrm{AD}, \mathrm{MCI}$ and healthy controls.

\footnotetext{
${ }^{1}$ Department of Computer Science, Pondicherry university, Pondicherry, India

${ }^{2}$ Department of Computer Science, Pondicherry university, Pondicherry, India
} 


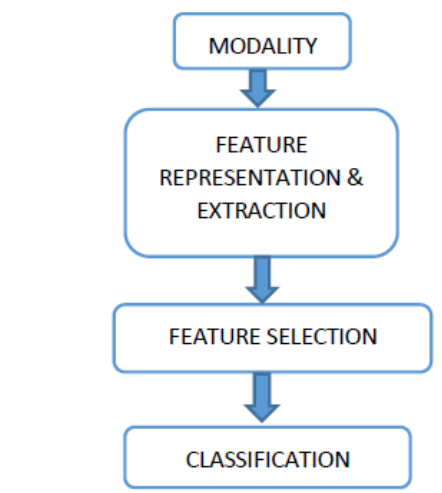

Fig 1. Basic Flow of classification of disease

The steps in Fig 1 shows the flow of the classification process dealt in the AD/MCI detection. The process starts from extracting the features, selecting and ending in classifying them.

\section{A. Feature-}

The images obtained from the modalities undergoes various steps of pre-processing and the features are extracted from them. A feature can be said as the distinctive attribute or characteristic that defines something. In this diagnosis model, features are the regions of brain images or Region of Interest(ROI) (for MRI and PET), the protein type (for CSF) or content, pattern or the gene (in case of genetic information). Even though many features are being extracted, only the top features that are affected by the disease are being selected for the classification.

Table-1 Top 11 brain regions selected as features for MCI classification from MRI and PET [29]

\begin{tabular}{cc}
\hline MRI & PET \\
\hline Amygdala right & Amygdala gyrus left \\
Hippocampal formation left & Precuneus left \\
Hippocampal formation right & Precuneus right \\
Uncus left & Inferior temporal gyrus left \\
Entorhinal cortex left & Anterior limb of internal capsule \\
right & Angular gyrus right \\
Amygdala left & Anterior limb of internal capsule \\
Middle temporal gyrus left & left \\
Temporal pole left & Global palladus left \\
Perirhinal cortex left & Globus palladus right \\
Uncus right & Posterior limb of internal capsule \\
& right \\
Para hippocampal gyrus left & Entorhinal cortex left \\
\hline
\end{tabular}

The features taken by CSF for AD/MCI classification:

- $\quad$ CSF A 442

- CSF t-tau

- $\quad$ CSF p-tau

The features taken by Genetic information for AD

- ApoE gene- $\varepsilon 2$

- ApoE gene- $\varepsilon 3$

- ApoE gene- $\varepsilon 4$

The selected features may undergo various other process for refinement and then undergoes the classification along with optimization process by using effective classifiers and machine learning techniques to classify between AD, MCI and Healthy subjects.

The features for the Computer aided diagnosis model are obtained from the modalities as images. The features are extracted from the images and represented according to the format applicable for the model. As a part of data pre-processing, removing noises, fusing data from different sources, normalization, data selection and reduction are done. The feature reduction helps in 
the selection and removal of unnecessary and redundant features. The feature reduction may be carried out as supervised or unsupervised manner. Dimensionality reduction must be done in such a way that it preserves the semantics and transforms the meaning of the data features for a good feature selection. The two main qualities of feature selection are relevancy and nonredundancy. Finally, the classification can be single-modal or multi-modal classification based on the number of modalities chosen for the model.

The image viewing software Dicom viewer can be used for viewing series of images obtained from the modalities and with Medical Image Processing, Analysis and Visualization (Mipav) software, the pre-processing part can be done. Tools used for dimensionality reduction are: Principal Component Analysis (PCA), Probabilistic PCA, Factor Analysis (FA), Classical multidimensional scaling (MDS), Sammon mapping, Linear Discriminant Analysis (LDA), Isomap, Landmark Isomap. Principal Component Analysis(PCA) is the most common tool used for dimensionality reduction. Lasso is one of the tool used for feature selection. The mostly used classification tool is SVM and its advanced version Multi-Kernel SVM. This has motivated researchers to propose a model with better accuracy.

\section{MODALITIES}

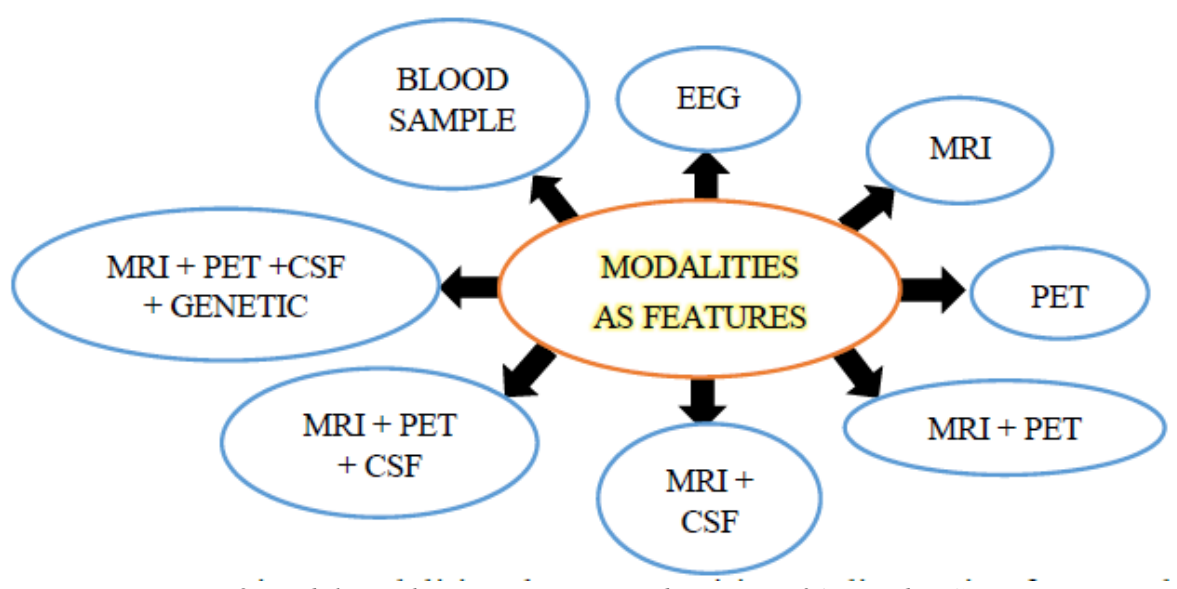

Fig 2 Modalities that are sensitive to diagnosis of $A D$ and $M C I$

The effectiveness of classification and prediction involves the modalities chosen for input and the methods used on it. The input must be chosen in such a way that it provides the essential information about the brain that is affected by dementia. Fig 2 shows many such modalities that screens the affected areas are in prevalence and is being used in the diagnosis of the disease. This survey focuses on the clinical modalities like Magnetic Resonance Imaging(MRI), Positron Emission Tomography (PET), Cerebrospinal Fluid(CSF), Blood samples, Electro -encephalogram(EEG), Genetic information and combinations are chosen for the diagnosis and classification of AD and MCI.

\section{A. Blood Sample}

Values of various protein content in blood samples helps in diagnosis of AD and MCI as the main symptom of this disease is the increase of protein content.

Lara Dantas and Mêuser Valença (2014) [4] proposed neural network models with Random Forest Algorithm on Multi-Layer Perceptron(MLP), Extreme learning Machine(ELM) and Reservoir Computing(RC). In this paper, they have created a new protein signature using the feature selection method in Weka InfoGainAttributeEva and provided results for the patient database used with a maximum of Reservoir Computing (93.22\%), Multi-Layer Perceptron (95.61\%) and Extreme Learning Machine (91.12\%).

\section{B. $E E G$}

Electroencephalography is a monitoring method to record the electrical activity of the brain to detect any brain disorders and the procedure consists of analysing the sleeping pattern of patients. EEG recordings are proved to be sensitive to the diseases. Francesco Carlo Morabito et al (2016) [18] proposed with EEG recordings that Deep Learning on Convolutional Neural Networks $(\mathrm{CNN})$ is used to generate features that can classify AD from MCI and from HC giving an average of $\mathbf{8 0 \%}$ correct classification by using a Multi layered Feedforward Perceptron (MLP).

\section{MRI}

Magnetic Resonance Imaging(MRI) is a medical imaging technique used to produce anatomy and physiological process of body with the use of magnetic field and radio waves. It also presents the pattern of grey matter in brain playing vital role in AD/MCI diagnosis. 
Esther E.Bron et al(2015)[2] proposed MRI based computed SVM weights as features. Feature selection based on SVM weights gave better results. Recursive feature elimination based on the p-map improved for AD-CN which increased from $90.3 \%$ without feature selection to $92.0 \%$.

Muhammad Aksan Iftikhar and Adnan Idris (2016]) [13] presents an ensemble classification framework, they combine thickness based features with volume based features of cortex thereby reducing features using F-Score feature selection method before classification. This method achieves accuracy of 91.66\%, 98.33\%, 90.83\% for AD - MCI, AD - NC, and NC MCI, respectively.

According to Klaus Fritzsche et al (2006) [7] a sequence of fully automated MRI-based image analysis to measure atrophy progression in brain. The use of ANNs, allows a better classification of the hidden data by a classical Fisher Linear Discriminant (FLD). The average sensitivity and specificity of the diagnosis of AD to be $81 \%$ and $70 \%$.

Emilie Gerardin et al (2009) [8] proposed Spherical Harmonics (SPHARM) coefficients to model hippocampi from MRI. It uses SPHARM-Point Distribution Model(SPHARM-PDM) with PCA, SVM and Bagging strategy for feature selection and leave-one out cross validation. It produces classification rate of $94 \%$ and $83 \%$ for $\mathrm{AD}$ v C and MCI v C respectively.

Chenhui Hu et al (2016) [11] proposed a paper with fMRI that uses deep learning in diagnosing brain diseases, AD/MCI and the functional connectivity between different brain regions. A targeted auto encoder network is built to classify the correlation matrix, which gives highest result with auto encoder of Time Series Data(TSD) $=67.50 \%$ and Correlation Coefficient data $(\mathrm{CCD})=87.50 \%$.

Lipeng Wang et al (2014) [26] proposed a connectivity network based framework to fuse multiple properties of network features with the fMRI as the imaging modality. It uses two network feature -brain regions and sub graph; Group analysis based - graph theoretical analysis is to differentiate between healthy and diseased, and machine learning based - features based on vectors extraction and classification. This paper achieved an accuracy of 97.27\%.

Jonathan H. Morra et al (2010) [19] proposed with T1 weighted MRIs to hippocampal segmentation to Machine Learning algorithms. It compared Ada SVM, Ada boost and free surfer where Ada SVM outperformed with precision in training:0.821(left) and0.844(right) and testing:0.785(left) and 0.802(right).

M.Rangini and Dr.G,Wiselin Jiji (2013)[20] discussed with T1 weighted brain MRIs. It compares two automated methods for hippocampal segmentation using Machine Learning algorithms: - SVM with manual Feature Selection and Hierarchical SVM with automated Feature Selection (Ada-SVM), and outperformed AdaBoost and Free surfer.

\section{PET}

Positron Emission Tomography is a nuclear imaging technique that produces images of glucose traces in brain and reduced glucose metabolism as Fluorodeoxyglucose-PET.

Eduardo Bicacro et al (2012) [1] proposed with PET, two alternative methods of feature extraction: 3D Haar-like features and histograms of gradient magnitude and orientation. For AD vs. CN classification, Haar-like features obtaining sensitivity 94.9\%, and the HGMO obtained highest mean accuracy $91.6 \%$.

Hossein Dehghan et al (2011) [6] proposed with F-FDG-PET that, the t-test, feature selection method which is commonly used in medical science, is not a sensitive measure, and Fisher linear discriminant ratio (FDR) is evaluated. Base on features extracted, a SVM differentiates normal control (NC) from AD or MCI with accuracy of $79.2 \%$ for NCvAD and $64.9 \%$ for NCvMCI.

Margarida Silveira and Jorge Marques (2010) [23] proposed with FDG-PET to boost classification by mixing simple classifiers which performs feature selection concurrently with segmentation which is well suited for high dimensionality problems, Boosting SVM(BSVM) with accuracies $90.97 \%, 79.63 \%$ and $70 \%$ for NC V AD, NC V MCI and MCI V AD respectively.

\section{E. MRI and PET}

Heung-Il Suk et al (2014) [24] proposed a high level feature representation for neuro imaging modalities like MRI and PET with deep learning and also Deep Boltzmann machine for fusing modalities. It uses patch extraction and patch level deep feature learning and produces accuracies of $95.35 \%, 85.67 \%$ and $74.58 \%$ for $\mathrm{AD} \mathrm{V}$ NC, MCI V NC and cMCI V ncMCI respectively.

Shuai Huang et al (2011) [12] proposed a sparse composite linear discriminant analysis model (SCLDA) for identifying disease-related brain regions from multi-modalities-MRI and PET. It identifies weaker features and allows joint analysis of features from multi-modalities. The classification accuracy was 94.3\% with Support Vector Machine(SVM).

Biao Jie et al (2015) [14] discussed about manifold regularized multitask feature learning method. It jointly selects the common features from multiple tasks. Finally, it uses the MKSVM to fuse multimodality data for classification and extending it to semi supervised, where only partial data are labelled with accuracies of $95.4 \%, 83 \%$ and $72.3 \%$ for AD-NC, MCI-NC and MCIc-MCInc respectively.

Feng Liu et al (2014) [16] introduced a method to evaluate using PET and MRI, it used both Diffusion Tensor Imaging (DTI) and resting-state functional MRI and obtained accuracy of $94.37 \%, 78.80 \%$ and $67.83 \%$ for AD-NC, MCI-NC and MCIcMCInc respectively. 
Siqi Liu et al (2015) [17] designed a framework with deep learning architecture AD diagnosis which uses a zero-masking strategy to extract complementary information from multiple data modalities -MRI and PET and fuse them with only less labelled data. It produces accuracies of $52.40 \%, 38.71 \%$ and $46.89 \%$ for $\mathrm{NC}, \mathrm{cMCI}$ and AD respectively.

Chen $\mathrm{Zu}$ et al (2016) [31] proposed a method that includes two subsequent components: label-aligned multi-task feature selection and multimodal classification with MRI and FDG-PET. The proposed method achieves better classification accuracy of $95.95 \%, 80.26 \%$ and $69.78 \%$ for AD-NC, MCI-NC and MCIc-MCInc respectively.

Chris Hinrichs et al (2011) [10] proposed a method based on Multi-Kernel Learning (MKL) framework, which outperformed an SVM trained on all available features by 3\%-4\%. It predicted with multi-modal disease marker (MMDM) conversion from $\mathrm{MCI}$ to AD and accuracy of $87.6 \%$ for AD-NC.

Yinghuan Shi et al (2014) [22] proposed a coupled feature representation of intra-coupled and inter-coupled interaction relationship with modalities MRI and PET. It proposed a coupled boosting algorithm that analyses the relation between modalities. It formulated a new weight updating function, which could perform on both incorrectly and inconsistently classified samples with an accuracy of $94.7 \%$ and $80.1 \%$ for AD V NC and MCI V NC respectively.

Jun Shi et al (2017) [21] discussed with MRI and PET a Deep Polynomial Network that performs well irrespective of the size of the datasets. It proposed multi modal stacked DPN(MMSDPN) to fuse and learn feature representation from multimodal neuroimaging data and also conducts both binary and multi modal classification. From the results, it was found that MMSDPN is better and produced accuracy for AD V NC :97.13\% using SVM classifier.

Tingting Ye et al (2015) [28] proposed a discriminative multi task feature selection, multi-modality based classification and inter-class and intra-class laplacian matrices with MRI and FDG-PET. It produced accuracies of AD v HC:95.92\%, MCI v HC: $82.13 \%$ and cMCI v ncMCI:71.12\%.

Xiao Zheng et al (2017) [30] introduced Learning Using Privileged Information(LUPI) algorithm with MRI as modality and PET as privileged information to produce RBM+ and SVM+. It proposed Multi modal Restricted Boltzmann Machine (MRBM) along with LUPI algorithm for effective feature representation as RBM+, SVM extended with LUPI classifier as SVM+, an ensemble LUPI algorithm for AD classification by integrating RBM+ and SVM+ and Multi Kernel Boosting(MKB) to boost the classification and integrate SVM+ and RBM+-SVM and produced accuracy :88.52\%.

Tien Duong $\mathrm{Vu}$ et al (2017) [25] proposed a deep learning method on fusing multimodalities like MRI and PET with Convolution neural network (CNN) trained and tested on combined PET-MRI data to diagnose the disease status of a patient. The accuracies are $91.14 \%(\mathrm{SAE}+\mathrm{CNN}), 91.1 \%(\mathrm{AD}-\mathrm{NC})$ and $89.2 \%(\mathrm{MCI}-\mathrm{NC})$.

\section{F. MRI and CSF}

Cerebrospinal fluid is a colourless fluid found in the brain and spine produced by choroid plexus and ventricles used as biomarker for quantification of specific proteins. The CSF A $\beta_{42}$, CSF t-tau and CSF p-tau are the features chosen for AD and MCI diagnosis.

Christos Davatzikos et al (2011) [5] proposed with MRI and CSF as biomarker a Spatial Pattern of Abnormalities for Recognition of Early $\mathrm{AD}$ (SPARE-AD) score and summarizing brain atrophy pattern for predictor of short term conversion with accuracy $55.8 \%$ for cMCI v ncMCI.

\section{G. MRI, PET and CSF}

Bo Cheng et al (2015) [3] proposed a model having domain transfer feature selection (DTFS) component, a domain transfer sample selection (DTSS), and a domain transfer support vector machine (DTSVM) with MRI, FDG-PET and CSF with a classification accuracy of $79.4 \%$ (cMCI-ncMCI)

Daoqiang Zhang et al (2011) [29] proposed to fuse three modalities, MRI, FDG-PET and CSF, to distinguish between AD (or $\mathrm{MCI}$ ) and healthy controls, using-MKSVM, multimodal data fusion and classification with accuracy of $93.2 \%$.

Xiaofeng Zhu et al (2014) [27] proposed a matrix similarity based loss function. It produces accuracies of $95.9 \%$ and $82 \%$ for AD V NC and MCI V NC respectively with MRI-PET-CSF and $93.8 \%$ for single modality.

Feng Li et al (2015) [15] proposed a learning system with MRI, PET and CSF using the dropout technique to avoid overfitting in deep learning. Stability selection and multitask learning strategy are added into the deep learning framework which achieved $91.4 \%, 77.4 \%, 70.1 \%$, and $57.4 \%$ accuracies for AD v HC, MCI v HC, AD v MCI, and MCI.C v MCI.NC classifications, respectively.

\section{H. MRI, PET, CSF and GENETIC INFORMATION}

The Alzheimer's disease is $70 \%$ caused by genes, so taking gene as feature would provide more accuracy in the prediction. The Apoliopoprotein (ApoE) gene $(\varepsilon 2, \varepsilon 3 \& \varepsilon 4)$ is associated with AD.

Katherine R.Gray et al(2013)[9] proposed a model with features such as regional MRI volumes, voxel-based FDG-PET signal intensities, CSF biomarker measures, and genetic information(ApoE). Based on the joint embedding, the method achieves classification accuracies of $89 \%$ between $\mathrm{AD} / \mathrm{HCs}$, and $75 \% \mathrm{MCI} / \mathrm{HCs}$ and $58 \%$ for cMCI / ncMCI. Random forests provide consistent pairwise similarity measures for multiple modalities, thus facilitating the combination of different types of feature data. 
Table-2 Comparison of the surveyed works grouped by modalities

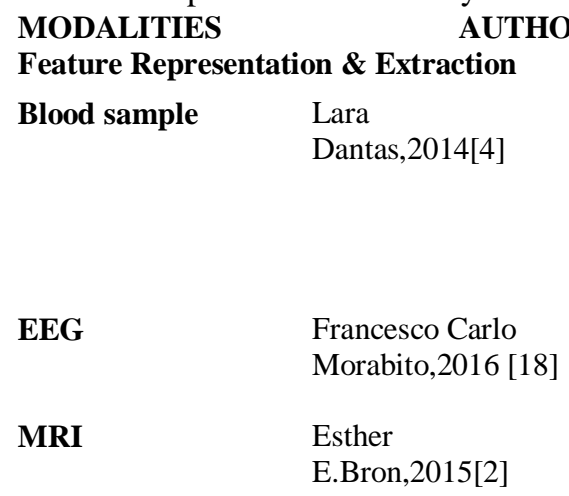

\begin{tabular}{|c|c|}
\hline $\begin{array}{l}\text { Muhammad Aksan } \\
\text { Iftikhar,2016[13] }\end{array}$ & $\begin{array}{l}\text { Cortical thickness, } \\
\text { volume hybrid based } \\
\text { features }\end{array}$ \\
\hline Klaus Fritzsche,2006[7] & ANN \\
\hline $\begin{array}{l}\text { Emilie Gerardin, } \\
\text { 2009[8] }\end{array}$ & SPHARM-PDM, \\
\hline Chenhui Hu,2016[11] & $\begin{array}{l}\text { Automated anatomical } \\
\text { labelled model, } \\
\text { representation method - } \\
\text { pearson's correlation } \\
\text { coefficient, }\end{array}$ \\
\hline Lipeng Wang, 2014[26] & $\begin{array}{l}\text { Connectivity network } \\
\text { based framework, } \\
\text { network features-brain } \\
\text { regions, subgraph }\end{array}$ \\
\hline $\begin{array}{l}\text { Jonathan H. } \\
\text { Morra,2010[19] }\end{array}$ & $\begin{array}{l}\text { Hippocampal } \\
\text { segmentation, }\end{array}$ \\
\hline $\begin{array}{l}\text { M.Rangini, } \\
\text { Dr.G,Wiselin } \\
\text { Jiji.,2013[20] }\end{array}$ & $\begin{array}{l}\text { Hippocampal } \\
\text { segmentation, } \\
\text { stereotaxic space, }\end{array}$ \\
\hline
\end{tabular}

METHODOLOGIES Feature selection ANN, RNN
Random forest
algorithm

\section{Significance map(p- map)-SVM weights(MRI) for SVM recursive feature limination ,SVM ignificance map,} Feature selection Ensemble classificationcoupled with ensemble classification, F-score feature selection

Bagging strategy $\quad$ SVM

p-value, recursive feature elimination

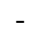

Support vector machines (SVM) with manual feature selection, hierarchical SVM with automated feature selection

\begin{tabular}{|c|c|c|c|}
\hline PET & $\begin{array}{l}\text { Eduardo } \\
\text { Bicacro,2012[1] }\end{array}$ & $\begin{array}{l}\text { 3D Haar like } \\
\text { features, Voxel } \\
\text { intensity features, } \\
\text { Histograms of } \\
\text { gradient magnitude } \\
\text { and orientation }\end{array}$ & PBCC \\
\hline $\begin{array}{l}\text { Hossein } \\
\text { Dehghan,2011[6] }\end{array}$ & $\begin{array}{l}\text { Montreal neurolog } \\
\text { imaging ,PCA }\end{array}$ & sical & \\
\hline $\begin{array}{l}\text { Margarida Silveira, } \\
\text { 2010[23] }\end{array}$ & Semi-automatic & - & \\
\hline MRI + PET & $\begin{array}{l}\text { Heung Il Suk, } \\
2014[24]\end{array}$ & $\begin{array}{l}\text { Patch extraction and } \\
\text { feature } \\
\text { representation }\end{array}$ & Patch-level \\
\hline
\end{tabular}

Shuai Huang,2011[12]

Biao Jie,2015[14]
Sclda, non-convex optimization, LDA parameter Atlas warping
L2/L1-regularizationmultitask feature selection Manifold regularized multi-task feature
Classification

ACCURACY

Multi layered perceptron, extreme learning machine reservoir computing

Deep learning on CNN -auto encoder with MLP

SVM $92.0 \%$ $80 \%$
$\mathrm{AD}: \mathrm{RC}(86.62 \%)$, $\operatorname{MCP}(93.44 \%)$, ELM (87.78\%); MCI:RC(69.29\%), $\operatorname{MCP}(68.15 \%)$, $\operatorname{ELM}(68.45 \%)$
SVM

Customised auto encoder, softmax

SVM

Fisher linear

discriminant

\section{MKSVM}

Ada Boost, Ada SVM, free surfer

Ada Boost, Ada SVM, free surfer

SVM, fisher linear discriminant Boosting SVM

Deep Boltzmann machine

SVM

Group sparsity regularizer, manifold
$\mathrm{AD}-\mathrm{MCI}=91.66 \%$; AD-NC $=98.33 \%$; NC-MCI $=90.83 \%$

$\mathrm{SEN}=81 \%$, $\mathrm{SPE}=70 \%$ $\mathrm{AD}$ v $\mathrm{C}=94 \%$, MCI v $\mathrm{C}=83 \%$

Time series data $=67.50 \%$.

Correlation coefficient data $=87.50 \%$

$97.27 \%$

Precision: training $=0.844$, testing $=0.802$ -
NC-MCI $=70.7 \%$; AD-NC $=88.1 \%$ $\mathrm{NC}-\mathrm{AD}=90.97 \%$, $\mathrm{NC}-\mathrm{MCI}=79.63 \%$, MCI-AD $=70 \%$ $\mathrm{AD}-\mathrm{NC}=95.35 \%$, MCI-NC $=85.67 \%$, cMCI$\mathrm{ncMCI}=74.58 \%$

$\mathrm{AD}-\mathrm{NC}=94.3 \%$ 


\begin{tabular}{|c|c|c|c|c|c|}
\hline & & & selection & $\begin{array}{l}\text { regularization-semi } \\
\text { supervised learning }\end{array}$ & MCIc-MCInc $=68.9 \%$ \\
\hline Feng Liu,2014[16] & \multicolumn{2}{|c|}{$\begin{array}{l}\text { Diffusion tensor imaging } \\
\text {,resting state functional } \\
\text { MRI }\end{array}$} & $\begin{array}{l}\text { Inter-modality } \\
\text { relationship constrained } \\
\text { multi-task feature } \\
\text { selection }\end{array}$ & MKSVM & $\begin{array}{l}\text { AD-NC }=94.37 \% ; \\
\text { MCI-NC }=78.80 \% ; \\
\text { cMCI-ncMCI }=67.83 \%\end{array}$ \\
\hline Siqi Liu,2015[17] & \multicolumn{2}{|c|}{ Zero masking strategy, } & - & $\begin{array}{l}\text { Stacked auto encoder, } \\
\text { MKSVM }\end{array}$ & $\begin{array}{l}\mathrm{NC}=52.40 \%, \\
\mathrm{cMCI}=38.71 \%, \\
\mathrm{ad}=46.89 \%\end{array}$ \\
\hline Chen $\mathrm{Zu}, 2016[31]$ & \multicolumn{2}{|l|}{-} & $\begin{array}{l}\text { Label aligned multi task } \\
\text { feature selection, }\end{array}$ & $\begin{array}{l}\text { Multi modal } \\
\text { classification, MKSVM }\end{array}$ & $\begin{array}{l}\text { AD-NC }=95.95 \% ; \\
\text { MCI-NC }=80.26 \% ; \\
\text { cMCI-ncMCI }=69.78 \%\end{array}$ \\
\hline Chris Hinrichs, $2011[10]$ & \multicolumn{2}{|l|}{$\begin{array}{l}\text { Voxel-based } \\
\text { morphometry }\end{array}$} & - & $\begin{array}{l}\text { Multimodal disease } \\
\text { marker, multi-kernel } \\
\text { learning, SVM, cross- } \\
\text { validation }\end{array}$ & $\mathrm{AD}-\mathrm{NC}=87.6 \%$ \\
\hline Yinghuan Shi, 2014[22] & \multicolumn{2}{|l|}{$\begin{array}{l}\text { Coupled feature } \\
\text { representation and } \\
\text { boosting }\end{array}$} & Lasso & SVM & $\begin{array}{l}\mathrm{AD}-\mathrm{NC}=94.7 \%, \\
\mathrm{MCI}-\mathrm{NC}=80.1 \%\end{array}$ \\
\hline Jun shi et al, 2017[21] & \multicolumn{2}{|c|}{$\begin{array}{l}\text { Deep polynomial } \\
\text { network, MMSDPN, } \\
\text { voxel based, vertex } \\
\text { based, ROI, patch-based }\end{array}$} & - & LC, SVM & $\mathrm{AD}-\mathrm{NC}=97.13 \%$ \\
\hline Tingting Ye[28] & \multicolumn{2}{|l|}{-} & $\begin{array}{l}\text { Discriminative multi } \\
\text { task feature selection, }\end{array}$ & $\begin{array}{l}\text { Multi-modality based } \\
\text { classification, inter- } \\
\text { class, intra-class } \\
\text { laplacian matrices, } \\
\text { random forest, MKSVM }\end{array}$ & $\begin{array}{l}\text { AD-HC }=95.92 \%, \\
\text { MCI-HC }=82.13 \%, \\
\text { cMCI-ncMCI }=71.12 \%\end{array}$ \\
\hline Xiao Zheng,2017[30] & \multicolumn{2}{|l|}{-} & - & RBM+,SVM+,LUPI & $88.52 \%$ \\
\hline $\begin{array}{l}\text { Tien Duong } \\
\mathrm{Vu}, 2017[25]\end{array}$ & \multicolumn{2}{|l|}{-} & - & $\begin{array}{l}\text { Deep learning method } \\
\text { on fused multi-modality } \\
\text { sparse auto encoder, } \\
\text { SVM }\end{array}$ & $91.4 \%$ \\
\hline MRI + CSF & $\begin{array}{l}\text { Christos } \\
\text { Davatzikos, 2011[5] }\end{array}$ & SPARE-AD & SPARE-AD & SVM & $\begin{array}{l}\text { cMCI- } \\
\text { ncMCI }=55.8 \%\end{array}$ \\
\hline MRI+ PET+CSF & Bo Cheng,2015[3] & PCA & DTFS, DTSS, & DTSVM & $79.4 \%$ \\
\hline $\begin{array}{l}\text { Daoqiang } \\
\text { Zhang,2011[29] }\end{array}$ & $\begin{array}{l}\text { direct feature } \\
\text { concatenation, }\end{array}$ & & - & $\begin{array}{l}\text { Multi modal data fusion } \\
\text { and classification-kernel } \\
\text { combination, MKSVM }\end{array}$ & $93.2 \%$ \\
\hline Xiaofeng Zhu, 2015[27] & - & & $\begin{array}{l}\text { Matrix similarity based } \\
\text { loss function - lasso }\end{array}$ & - & $\begin{array}{l}\text { AD-NC }=95.9 \%, \\
\text { MCI-NC }=82 \%,\end{array}$ \\
\hline Feng Li,2015[15] & PCA & & $\begin{array}{l}\text { PCA, stability selection, } \\
\text { dropout and MTL }\end{array}$ & SVM & $\begin{array}{l}\text { AD-HC=91.4\%; } \\
\text { MCI-HC }=77.45 ; \\
\text { AD-MCI }=70.1 \% ; \\
\text { cMCI-ncMCI }=57.4 \%\end{array}$ \\
\hline $\begin{array}{l}\text { MRI+PET+CSF } \\
\text { +Genetic }\end{array}$ & $\begin{array}{l}\text { Katherine } \\
\text { R.Gray,2013[9] }\end{array}$ & ApoE g & Random forest & - & $\begin{array}{l}\text { AD-HC }=89 \% \\
\text { MCI-HC }=75 \% \\
\text { cMCI-ncMCI }=58 \%\end{array}$ \\
\hline
\end{tabular}

The survey has been done with the modalities as input and various researchers have proposed models with different techniques for each step of classification. The techniques that has been used for feature extraction and representation are Artificial Neural Networks(ANN), Recurrent Neural Networks(RNN), Recursive feature elimination, hybrid based features, Spherical harmonics -Point distribution model(SPHARM-PDM), Automated Anatomical labelled(AAL) model, Pearson's correlation co-efficient, Connectivity network based framework, network features-brain regions, subgraph, Hippocampal segmentation, diffusion tensor imaging, zero masking strategy, Haar like features, Voxel intensity features, Histograms of gradient magnitude and orientation, PCA etc., The techniques used for feature selection are Random forest, bagging strategy, f-score, recursive elimination, patch-level, stability selection, manifold regularized multi task, DTFS, DTSS etc., The techniques used for classification are SVM, MLP, ELM, RC, fisher linear discriminant, softmax, MKSVM, Ada Boost, Ada SVM, Freesurfer, random forest, DTSVM, SVM+, RBM+ etc.,

The survey of research works has been tabulated with the headings such as author, modalities, methodologies and its accuracy found in each work and are grouped according to the modalities used in it. From the above table, the techniques used for the steps of diagnosis- Feature representation and extraction, feature selection and classification, and the accuracies of each work can be easily referred. From the table, the MRI Imaging modality has been recognized to be sufficient enough for classification, if efficient techniques have been imposed on it, producing AD-MCI=91.66\%; AD-NC=98.33\%; NC- 
MCI $=90.83 \%$ [13] and adding of more modalities may increase the accuracy. Support Vector Machine(SVM) has been the efficient classifier used commonly, but it has a developed version, Multi-kernel SVM(MKSVM). The future works can gain a comparison from the above survey and propose a more efficient combination.

\section{CONCLUSION:}

The choice of modalities is an important task in the prediction and classification of Alzheimer's disease and Mild Cognitive Impairment. It gives the essential features needed for accurate diagnosis. But the choice of methods and tools for Feature extraction, representation and classification decides the accuracy level of model. Thus a new model can be proposed with effective tools for each step of classification to bring more performance in the diagnosis.

\section{REFERENCES:}

[1] Eduardo Bicacro , Margarida Silveira , Jorge S . Marques ," Alternate Feature Extraction Method in 3D Brain Image-Based Diagnosis Of Alzheimer's Disease", 1237-1240, 2012.

[2] Bron, E. E., Smits, M., Niessen, W. J., \& Klein, S.. Feature Selection Based on the SVM Weight Vector for Classification of Dementia, 2015.

[3] Cheng, B., Liu, M., Zhang, D., Munsell, B. C., Shen, D., \& Member, S. ,"Domain Transfer Learning for MCI Conversion Prediction", 2015.

[4] Dantas, L. ,"Using neural networks in the identification of signatures for prediction of Alzheimer' s Disease",2014.

[5] Davatzikos, C., Bhatt, P., Shaw, L. M., Batmanghelich, K. N., \& Trojanowski, J. Q." Prediction of MCI to AD conversion, via MRI , CSF biomarkers , and pattern classification.", 2011.

[6] Dehghan, H., Pouyan, A. A., \& Hassanpour, H. ,"SVM-based Diagnosis of the Alzheimer's Disease Using 18F-FDG PET with Fisher Discriminant Rate", 14-16, (December), 2011.

[7] Fritzsche, K., Wangenheim, A. Von, \& Unterhinninghofen, R.," Automated MRI-Based Quantification of the Cerebral Atrophy Providing Diagnostic Information on Mild Cognitive Impairment and Alzheimer's Disease". 2006.

[8] Gerardin, E., Chételat, G., Chupin, M., Cuingnet, R., \& Desgranges, B.," NeuroImage Multidimensional classification of hippocampal shape features discriminates Alzheimer's disease and mild cognitive impairment from normal aging. NeuroImage, 1476-1486, 2009.

[9] Gray, K. R., Aljabar, P., Heckemann, R. A., \& Hammers, A. ,"NeuroImage Random forest-based similarity measures for multi-modal classi fi cation of Alzheimer's disease. NeuroImage", 65, 167-175,2013.

[10] Hinrichs, C., Singh, V., Xu, G., \& Johnson, S. C. ,"NeuroImage Predictive markers for AD in a multi-modality framework : An analysis of MCI progression in the ADNI population". NeuroImage, 574-589, 2011.

[11] Hu, C., Ju, R., Shen, Y., Zhou, P., \& Li, Q.," Clinical Decision Support for Alzheimer 's Disease Based on Deep Learning and Brain Network. 2016.

[12] Huang, S., Li, J., Ye, J., Chen, K., Wu, T., \& Fleisher, A.," Identifying Alzheimer' s Disease-Related Brain Regions from Multi-Modality Neuroimaging Data using Sparse Composite Linear Discrimination Analysis", 1-9, 2011.

[13] Iftikhar, M. A., \& Idris, A.," An Ensemble Classification Approach for Automated Diagnosis of Alzheimer 's Disease and Mild Cognitive Impairment", $78-83,2016$.

[14] Jie, B., Zhang, D., Cheng, B., Shen, D., Hill, C., \& Carolina, N.," Manifold Regularized Multitask Feature Learning for Multimodality Disease Classification" , 489-507,2015.

[15] Li, F., Tran, L., Thung, K., Ji, S., Shen, D., \& Li, J. "A Robust Deep Model for Improved Classification of AD / MCI Patients", 1610-1616, 2015.

[16] Liu, F., Wee, C., Chen, H., \& Shen, D. "NeuroImage Inter-modality relationship constrained multi-modality multi-task feature selection for Alzheimer ' s Disease and mild cognitive impairment identification". NeuroImage, 466-475, (2014).

[17] Liu, S., Member, S., Liu, S., Member, S., Cai, W., Che, H., ... Fulham, M. J. ,"Multimodal Neuroimaging Feature Learning for Multiclass Diagnosis of Alzheimer's Disease", 1132-1140, 2015.

[18] Morabito, F. C., Ieracitano, C., Bonanno, L., Bramanti, A., De, S., \& Bonino-pulejo, I. C. N.," Deep Convolutional Neural Networks for Classification of Mild Cognitive Impaired and Alzheimer's Disease Patients from Scalp EEG Recordings" 2016.

[19] Morra, J. H., Tu, Z., Apostolova, L. G., Green, A. E., Toga, A. W., \& Thompson, P. M.," Comparison of AdaBoost and Support Vector Machines for Detecting Alzheimer's Disease Through Automated Hippocampal Segmentation",30-43, 2010.

[20] M.Rangini , Dr.G,Wiselin Jiji. ,"Detection of Alzheimer's Disease Through Automated Hippocampal Segmentation", 144-149. 2013.

[21] Shi, J., Zheng, X., Li, Y., Zhang, Q., Ying, S., \& Member, I. ,"Multimodal Neuroimaging Feature Learning with Multimodal Stacked Deep Polynomial Networks for Diagnosis of Alzheimer' s Disease", 2017.

[22] Shi, Y., Suk, H., Gao, Y., \& Shen, D. ,"Joint Coupled-Feature Representation and Coupled Boosting for AD Diagnosis",2014.

[23] Silveira, M., \& Marques, J.," Boosting Alzheimer Disease Diagnosis using PET images.",2010.

[24] Suk, H., Lee, S., Shen, D., \& Initiative, N. ,"NeuroImage Hierarchical feature representation and multimodal fusion with deep learning for AD / MCI diagnosis". NeuroImage. 2014.

[25] Vu, T. D., Yang, H., Nguyen, V. Q., Oh, A., \& Kim, M. ,"Multimodal learning using Convolution Neural Network and Sparse Autoencoder", 309-312, 2017.

[26] Wang, L.," Combining Multiple Network Features for Mild Cognitive Impairment Classification",2014.

[27] Xiaofeng Zhu, Heung-Il Suk, "Multi-Modality Canonical Feature Selection for Alzheimer's Disease Diagnosis," Springer Int. Publ., vol. 17, no. 2, pp. $162-169,2015$.

[28] Ye, T., Zu, C., Jie, B., Shen, D., \& Zhang, D.," Discriminative Multi-Task Feature Selection for Multi-modality Based AD / MCI Classification", 15-18, 2015.

[29] Zhang, D., Wang, Y., Zhou, L., Yuan, H., \& Shen, D.," NeuroImage Multimodal classi fi cation of Alzheimer' s disease and mild cognitive impairment". NeuroImage, 856-867, 2011.

[30] Zheng, X., Shi, J., Zhang, Q., Ying, S., \& Li, Y. (2017). Improving MRI-Based Diagnosis Of Alzheimer's Disease Via An Ensemble Privileged Information Learning Algorithm, School of Communication and Information Engineering, Shanghai University, China Department of Mathematics , School of Science, Shanghai University, China Shenzhen City Key Laboratory of Embedded System Design , College of Computer Science and Software Engineering, Shenzhen University, China, (2), 456-459.

[31] Zu, C., Jie, B., Liu, M., Chen, S., \& Shen, D. (2016). Label-aligned multi-task feature learning for multimodal classification of Alzheimer 's disease and mild cognitive impairment. Brain Imaging and Behavior, 1148-1159. https://doi.org/10.1007/s11682-015-9480-7 